\title{
Automated Particle Collection for Protein Crystal Harvesting
}

\section{Journal Article}

Author(s):

Zeydan, Burak; Petruska, Andrew J.; Somm, Luca; Pieters, Roel; Fang, Yang; Sargent, David F.; Nelson, Bradley J.

Publication date:

2017-07

Permanent link:

https://doi.org/10.3929/ethz-b-000167454

Rights / license:

In Copyright - Non-Commercial Use Permitted

Originally published in:

IEEE Robotics and Automation Letters 2(3), https://doi.org/10.1109/LRA.2017.2669364

Funding acknowledgement:

665718 - Automated Microhandling System for X-ray Crystallography (EC) 


\title{
Automated Particle Collection for Protein Crystal Harvesting
}

\author{
Burak Zeydan, Andrew J. Petruska, Luca Somm, Roel Pieters, Yang Fang, David F. Sargent, and \\ Bradley J. Nelson
}

\begin{abstract}
A robust automated system to collect protein crystals for X-ray crystallography is presented. This system uses an ultra violet imaging system based on commercial off the shelf components, a magnetically manipulated tool, and a resilient behavior-based controller. The system is validated by collecting over 350 polystyrene beads, used as crystal emulators, and transporting them $2 \mathrm{~mm}$ to a predefined goal in a 14 hour period without human intervention. The average time to identify, collect, transport, and deliver a crystal emulator is 2.4 minutes, similar to an expert operator. This is the first demonstration of a completely automated robust system for protein crystal harvesting.
\end{abstract}

\section{INTRODUCTION}

Modern pharmaceutical engineering often uses the physical protein structure to understand the interaction between a potential drug and its intended target. This structure can be obtained using X-ray crystallography. The basic process is described in [1] as follows. First, well-ordered crystals are nucleated and grown in individual containers under highly controlled conditions. Once mature, the crystals are removed from the growth medium (harvested), cryoprotected, and flash-cooled. Finally, the crystals are placed into an X-ray beam and their diffraction pattern is recorded and numerically analyzed to reconstruct the underlying physical structure. Due to the high demand of this technique, most of these steps have been both highly parallelized and automated. However, one of the most laborious processes, the actual harvesting of the crystals, is still performed manually.

To harvest crystals, operators scoop the crystals out of their growth medium with micro-loops while observing the procedure under magnification, see Fig. 1. This slow and laborious process can take an expert operator on average 2.4 minutes per crystal [2]. Further compounding this task, the size range of the crystals is on the order of hand tremor $(50 \mu m$ [3]). This tremor can cause the operator to inadvertently damage a crystal while harvesting and render it unusable. Together, the slow harvesting rate and the high loss rate make crystal harvesting the major bottleneck of highthroughput crystallography [2]. Many attempts to automate or

B. Zeydan, L. Somm, R. Pieters, Y. Fang, A. J. Petruska, D. Sargent, and B. J. Nelson are with ETH Zurich, Switzerland. A. J. Petruska is now with the Colorado School of Mines, USA. R. Pieters is now with Aalto University, Finland. E-mail: zeydanb@ethz.ch, apetruska@mines.edu, sargent@mol.biol.ethz.ch, roel.pieters@aalto.fi, bnelson@ethz.ch. The work was partially supported by the European Research Council Proof of Concept grant RODBOT.

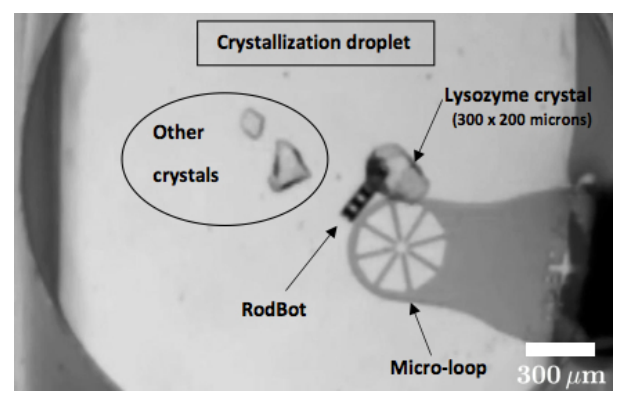

Fig. 1. Microscope view of the crystal harvesting operation. The lysozyme crystal is transported towards the micro-loop for extraction.

semi-automate this procedure have been pursued (see [2] for a comprehensive review). Although many advancements have been made, the two main technical challenges to harvesting, namely real-time crystal identification and segmentation, and robust and precise crystal manipulation, have yet to be effectively combined in one system. Systems making use of microcapillaries [4], [5], microgrippers [6], [7], acoustic tweezers [8], optical tweezers [9], and microrobots [10], [11] are attempts at semi-automating the process, delegating the challenging tracking and manipulation planning to operators.

Automated crystal segmentation is challenging because crystals do not share shape or edge related features [2]. Not only is this true from one crystal to another, but also for an individual crystal when viewed during manipulation from changing perspectives [2]. Early efforts to detect and segment crystals used the visible light spectrum [12]-[14], but were not robust. Imaging techniques that do not rely on the human-observable spectrum [15]-[17] have proven to be more reliable for crystal detection. Unfortunately, the cost and lack of external control limits their applicability to automated harvesting systems.

Robust and delicate micromanipulation has been the subject of numerous investigations, see for example [18] for a general overview. However, robust automatic control in the context of micro-scale manipulation and assembly remains an open question. Thakur et. al recently proposed a controller with three operation modes for optical tweezer based manipulation of cells. At any moment, one of the push, align, and backup control maneuvers is executed according to a threshold based feedback policy algorithm where the thresholds are determined through a genetics algorithm in a reliable simulation environment [19]. Although this represents a significant step, there is no work that has achieved 
the sort of automatic planning that has been mentioned by Firby [20] at the micro-scale.

Here we propose a system to automate the harvesting step of X-ray crystallography. Robust segmentation is achieved through an ultraviolet (UV) imaging system comprising readily available and affordable off-the-shelf components. It is described in more detail in Section II-B. Robust planning and control is achieved by a reactive behavior-based planner, an approach similar to [19], and is discussed in Section II-C. To execute these behaviors, low level control will be completed with the methods described in [21]. The capabilities of the resulting system is demonstrated in Section III. Finally, Section IV discusses the results and conclusion.

\section{Materials AND Methods}

\section{A. Manipulation Hardware}

Crystals are manipulated with a microrobotic device called the RodBot, which is a magnetically manipulated mobile device with a typical cross section of $50 \times 60 \mu \mathrm{m}$ and a length of 300 to $600 \mu \mathrm{m}$. It is designed as a rectangular polymer (SU8) rod with internal, transverse soft-magnetic posts consisting of a cobalt-nickel (CoNi) alloy. These posts align with an external magnetic field enabling rolling propulsion. For more information on the design, modeling, and control of a RodBot see [10], [11], [21].

The magnetic field used to manipulate the RodBot is generated by an eight-coil Magnetic Field Generator $\left(\mathrm{MFG}^{1}\right)$ (see Figure 2-1) [22]. The system is capable of generating magnetic fields and field gradients up to $20 \mathrm{mT}$ and $2 \mathrm{~T} / \mathrm{m}$ at control frequencies up to $2 \mathrm{kHz}$ [23]. This system is capable of 5 degree of freedom (DOF) wireless control of magnetic micro- and nano-structures [24]. We used this device to generate $3 \mathrm{D}$ magnetic fields to induce $2 \mathrm{D}$ motion on the agent as described in [11].

The MFG and RodBot provide precise control of the crystals, which can be observed with moderate magnification. To extend the manipulation area beyond the magnetic workspace of the MFG and observable workspace of the objective lens, a coarse XY stage (ASR100B120B-E03T3, Zaber) is used (see Figure 2-3). This stage moves the wellplate that contains the crystals in solution and the RodBot and keeps the manipulation region of interest centered in the manipulation system. Since the forces at the length scale of the RodBot are dominated by Stokes drag and van der Waals interactions, the slight inertial forces generated by the stages motion do not appreciably affect the position of the RodBot or crystals relative to their fluidic environment. The XY stage increases the achievable workspace to $100 \times 120 \mathrm{~mm}$. The system is shown in Figure 2.

\section{B. UV Imaging and Crystal Detection}

Segmentation of the protein crystals exploits the autofluorescence of Tryptophan, a common amino acid in the crystals.

\footnotetext{
${ }^{1}$ http://www.magnebotix.com
}

Tryptophan has an absorption peak at $280 \pm 20 \mathrm{~nm}$ [25] and emits fluorescence light at $350 \pm 50 \mathrm{~nm}$ [26]. This unique signature allows the crystals to be easily distinguished from their background as shown in [27]. The auto-fluorescence properties of SU-8, especially in the near-UV range, also enables the detection of the RodBot with the same optical system, see Fig. 3. Fluorescence imaging equipment for crystal segmentation are commercially available, however they come with significant cost and are not designed to be integrated into a control system. Fortunately, it is possible to assemble a custom system with commercial off-the-shelf (COTS) components that is both cost effective and easily integrated into the control architecture.

The optical system depicted in Fig. 3 consists of a UV LED source (M280L3, Thorlabs), collimating objective (87-979, Edmund Optics), diffuser (DGUV10-600, Thorlabs), beamsplitter (SEM-FF310-Di01-25x36, Semrock), magnification objective (LMU-10X-UVB, Thorlabs), UV filter (BP324, Midwest Optical Systems), and camera (scA640-120gm, Basler). The camera selected is unique as its imaging sensor (Sony ICX618) is compatible with imaging near-UV light. The total cost of the system is 5100 USD, which includes the camera (621 USD), the objective (1712 USD), the beamsplitter (605 USD), the LED light source (1498 USD), and various lenses, tubes, and filters required for assembly (671 USD). Compared to the cost of a specialized UV camera of 10000-20000 USD, our system is significantly less expensive. The system functions as follows. The LED emits light at $\sim 280 \mathrm{~nm}$, this light is collimated by a lens, diffused, and directed towards the beam splitter which transmits light at wavelengths greater than $315 \mathrm{~nm}$ and reflects light at wavelengths of $275 \pm 20 \mathrm{~nm}$ to illuminate protein crystals. Once exposed, the crystals fluoresce $350 \mathrm{~nm}$, which is transmitted by the beam splitter towards the camera, filtered with a bandpass filter allowing only $350 \pm 30 \mathrm{~nm}$ to prevent extraneous light in the visible spectrum from exciting the camera sensor, and focused onto the camera's sensor by

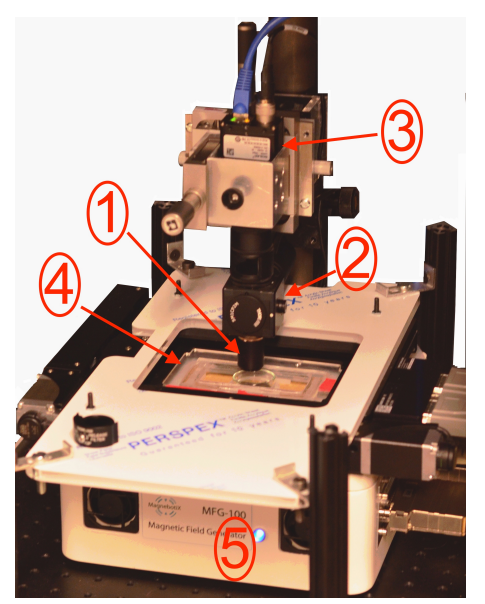

Fig. 2. Overview of the system. The UV-system consisting of the objective (1), the beam splitter (2), and the camera (3), can be seen. The XY stage (4) is mounted on top of the Magnetic Field Generator (5) (MFG). 


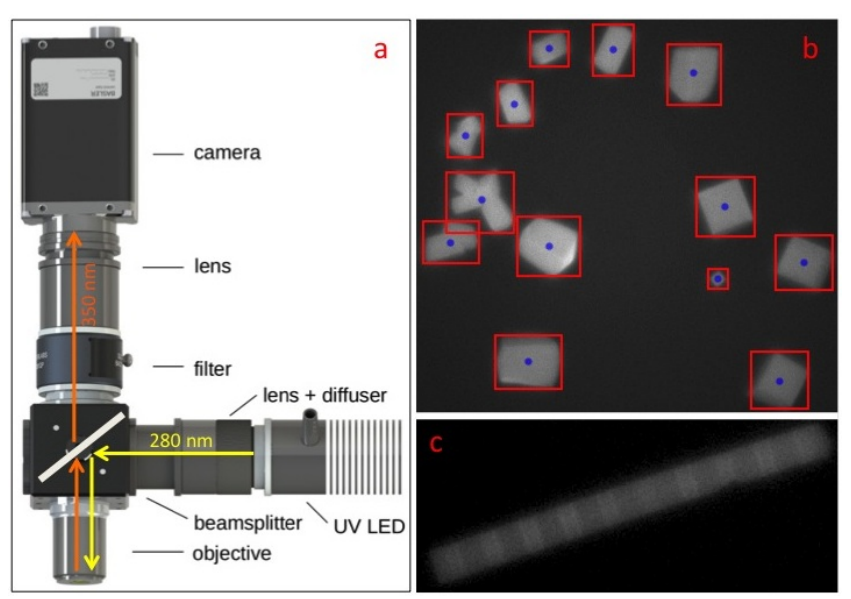

Fig. 3. The optical system (a) consists of a UV LED source, appropriate lenses, beamsplitter and objective for light path guidance, filters for desired wavelength transmissions and a camera for imaging of the desired wavelength of the UV signal. Protein crystal fluorescence and the detection accuracy can be seen in (b). Crystals as small as $30 \mu \mathrm{m}$ can be detected accurately. The RodBot fluorescence is shown in (c).

a tube lens (65-976, Edmund Optics) to form an image. The objective used has a magnification of $10 \mathrm{X}$ offering a $1.52 \mathrm{~mm} \times 1.14 \mathrm{~mm}$ field-of-view which is recorded by the digital camera at a resolution of $659 \times 494 \mathrm{px}$ (corresponding to a pixel size of $5.6 \mu \mathrm{m} \times 5.6 \mu \mathrm{m}$ ). Finally, an Arduino module is used to control the triggering of the LED. Since both the SU-8 and the crystals bleach when exposed to too much light, it is critical to control the total exposure time used throughout the procedure. Fig. 3b shows the optical results from our imaging system. Comparing Fig. 3b with Fig. 1, it is clear that the contrast is much higher than what is recorded with traditional imaging techniques.

Once these high contrast images are recorded, the computer then identifies and segments the crystals and the RodBot. This is performed as follows. First, a Canny edge detector is used to extract the outlines of potential crystals based on the intensity gradient. The image is then dilated to form closed curves, and a filling operation transforms the curves into blobs. Finally, blob detection [28] returns the center, size and circularity of the extracted crystals. These measures are subsequently used to assess the image and the individual blobs for classification.

\section{Planning Architecture}

In a complex and highly variable Environment, robustness can be achieved with an equally complex controller based on a high fidelity model, or by a controller that is designed to recover gracefully when its underlying model fails to predict the system well. Here we choose the latter. To achieve this corrective behavioral capability, a behaviorbased planner inspired by Firby's [20] and Brooks' subsumption architecture [29] is developed. Behavior-based planners decompose the control task into a set of activities [30]. Each of these activities is associated with its own control strategy. Planners of this kind are being used in a wide range of applications including, music improvisation [31], flight control [32], [33], emergency obstacle avoidance [34], and intelligent transportation systems [35]. For crystal harvesting with a RodBot, these activities can be enumerated as

Protect System Monitor all devices and stop operation if performance measures (e.g., temperature) leave acceptable operational ranges.

Map Workspace Initial scan of the workspace with XY stage to identify the RodBot, crystals, and drop-off locations.

Center Image Modify the position of the XY stage to keep the RodBot approximately centered in the optical field of view.

Avoid Obstacle Actively steer the RodBot magnetically around an obstacle using a potential-field-like approach [36] (e.g., a crystal not targeted for harvesting) [21].

Select Crystal Automatic selection of the target crystal based on quality metrics such as size.

Approach Crystal Magnetically maneuver the RodBot to the target crystal location [21].

Pickup Crystal Control the RodBot's motion to lift the target crystal into the RodBot's fluidic vortex trap [21].

Carry Crystal Maneuver the RodBot and carry crystal towards the drop off location [10].

Drop Crystal Control the RodBot's motion to release the crystal from the fluidic vortex trap [21].

Resembling the implementation in [37], our implementation consists of four main entities: 1) a set of $n$ behaviors $B_{q}=\left\{b_{i} \mid i \in[1, n]\right\}$ 2) the list of $n_{d}$ devices $D_{q}=$ $\left\{d_{i} \mid i \in\left[1, n_{d}\right]\right\}$ containing all the sensors and actuators in the system, 3) a state $s$ that contains the sensor data from the previous measurements, and 4) a list of $n_{r}$ rules $R_{q}=\left\{r_{i} \mid i \in\left[1, n_{r}\right]\right\}$ bringing together all the decision thresholds and execution parameters for all the behaviors. Each behavior implements the following set of functions:

- $\operatorname{void} \operatorname{Setup}\left(R_{q}\right)$ : initializes the behavior and the rules that it uses.

- bool ShouldRun $\left(R_{q}, D_{q}, s\right)$ : takes the state and the lists of rules and devices and determines if a behavior should run. If a part of the state information is considered outdated or inaccurate according to the rules, this function can access the sensors and actuators to acquire the additional necessary information.

- $\operatorname{void} \operatorname{Run}\left(R_{q}, D_{q}, s\right)$ : takes the the state and a list of rules and devices and executes the desired action.

The algorithm operates as follows:

Here the behaviors are ordered according to their priorities in $B_{q}$. If a high priority behavior should become active, then it takes over the control, or subsumes, the currently active behavior. Crystals are sensitive to UV exposure and can easily become damaged. In order to alleviate the risk, our behaviors take images infrequently at an average rate of 0.5 frames-perseconds, and the most up-to-date information is summarized in the state $s$. 


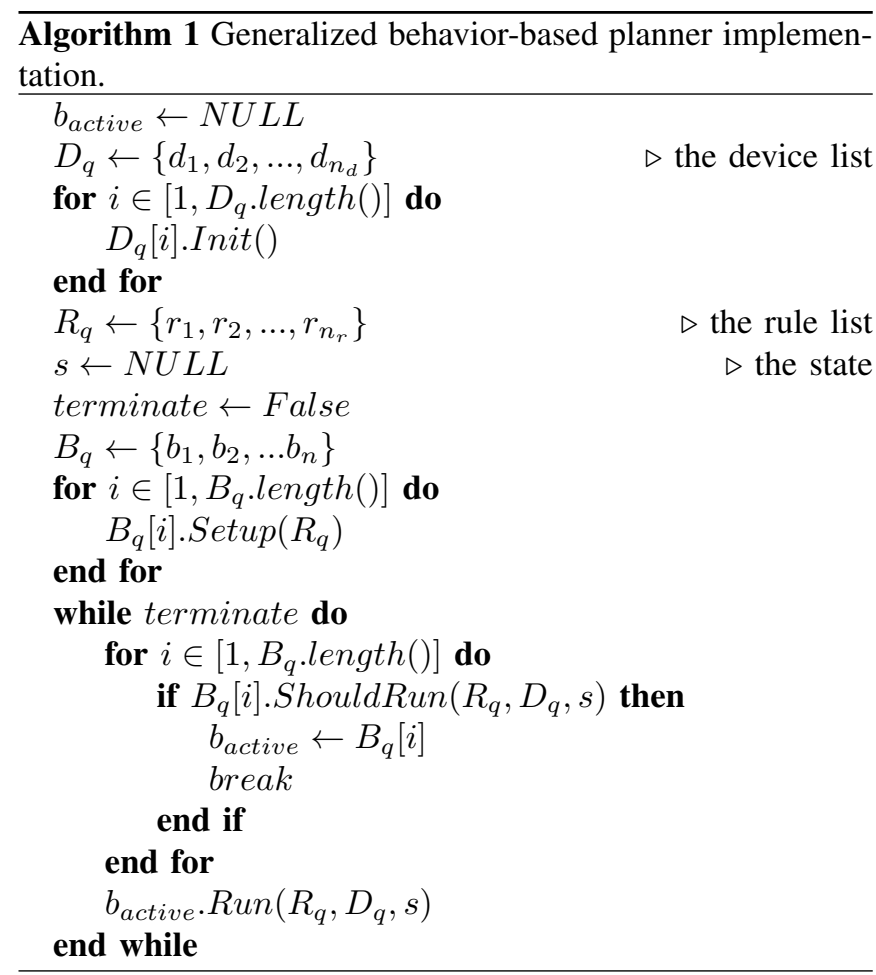

\section{Computational Hardware}

The system is controlled by a desktop computer (Intel Core i5 quad core $3.20 \mathrm{GHz}$ CPU, 8 GB RAM), running 64bit Ubuntu 12.04. The programming is done in MATLAB. The low level functionalities including image processing and sending commands to the MFG are implemented in $\mathrm{C} / \mathrm{C}++$ and compiled as functions to be called from MATLAB. The presented behavior based planning algorithm is implemented fully in MATLAB.

\section{EXPERIMENTAL VALIDATION}

\section{A. Crystal Damage Due to UV Exposure}

The effect of UV on the crystals is investigated by continuously exposing a lysozyme crystal $c_{1}$ with UV over a period of 10 minutes, taking an image every minute. Manually selecting an image patch $C$ with $n_{i m}$ pixels on the crystal and a background patch $B$ with $n_{b g}$ pixels, a signal-to-noise-ratio $S N R$ metric is calculated for each image according to

$$
S N R=\frac{\frac{1}{n_{i m}} \sum_{i=0}^{n_{i m}} C(i)-\frac{1}{n_{b g}} \sum_{j=0}^{n_{b g}} B(j)}{\frac{1}{n_{b g}} \sum_{j=0}^{n_{b g}} B(j)} .
$$

As a control, the image of another lysozyme crystal $c_{2}$ is acquired by exposing it to UV only during the image acquisition. Results are presented in Figure 4. As can be seen, while the SNR for $c_{2}$ decreased by only $4 \%$, it decreased by $58 \%$ for $c_{1}$.

In [38] this considerable decay in SNR is associated with 1) structural damage at a molecular level due to photoionization in the form of breaking covalent bonds; or to
2) protein degeneration due to local heating of samples. As analyzed in [39], in order to avoid radiation damage, UV exposure should be limited to 1 second with $260 \mathrm{~nm}$ light, corresponding to $6.75 \times 10^{15} \frac{\text { photons }}{\mathrm{mm}^{2}}$ or $5 \frac{\mathrm{mW}}{\mathrm{mm}^{2}}$. The estimated effective power output $P_{\text {eff }}$ of our system is $5 \mathrm{~mW}$. Considering the measured LED spot size on the sample $A_{s s}$ of $100 \mathrm{~mm}^{2}$, the power density $\frac{P_{e f f}}{A_{s s}}$ is calculated to be $0.05 \frac{\mathrm{mW}}{\mathrm{mm}^{2}}$. So, with our $280 \mathrm{~nm}$ LED source, the total UV exposure of the crystals should be limited to $100 s$ to avoid significant damage, which is consistent with our test.

\section{B. Crystal Detection and Tracking}

The performance of crystal detection is evaluated on 120 different samples containing a total of 215 lysozyme crystals with sizes raging between $30 \mu \mathrm{m}$ and $300 \mu \mathrm{m}$. There were a maximum of 12 crystals per sample. The accuracy is defined by the ratio of correctly detected wells to the total number of wells. For a well to be classified as correctly detected, the crystals identified must match the human evaluation. Detection performance is evaluated for several camera parameter sets, and an accuracy of $98.3 \%$ is achieved for a camera gain of 500 and an exposure time of $3 \mathrm{~ms}$. Overall the detection failed in only two cases: 1) when two crystals were too close so that the boundary between them was barely visible, and 2 ) in the presence of an artifact in the well.

\section{Harvesting Task}

Protein crystals are very sensitive to changes in medium conditions; therefore, to perform long duration continuous experiments $105-125 \mu \mathrm{m}$ polystyrene beads are used to mimic the crystals, as was done in [4], [6]. As the interaction between the RodBot and crystals with various types, shapes, and sizes has been thoroughly investigated and it has been shown that a tele-operated RodBot can successfully perform the harvesting task [11], the substitution of the beads for crystals will not affect the applicability of the experiment's results. Fortunately, the polystyrene beads autofluoresce at

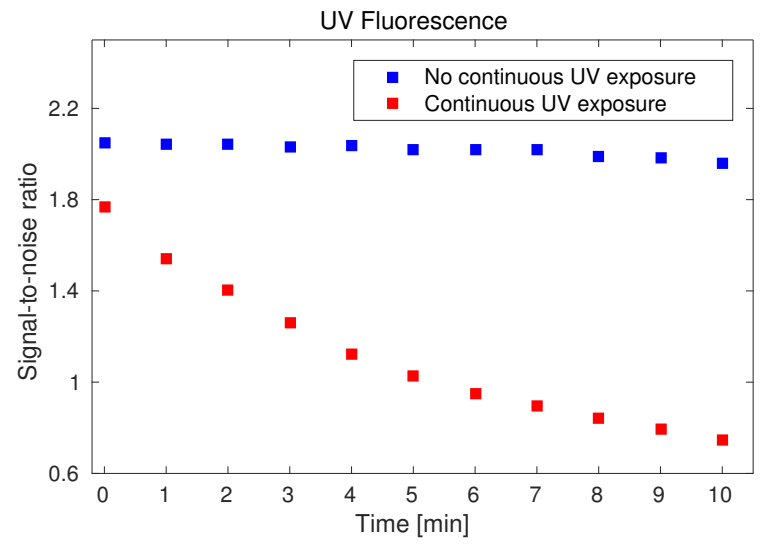

Fig. 4. Protein crystals bleach when continuously exposed to UV radiation. (blue) the fluoresce SNR of a crystal exposed to UV only during imaging. (red) the SNR of a crystal continuously exposed to UV. As can be seen from the graph, the total exposure time should not exceed 100 seconds to keep the SNR ratio above 1.5 . 


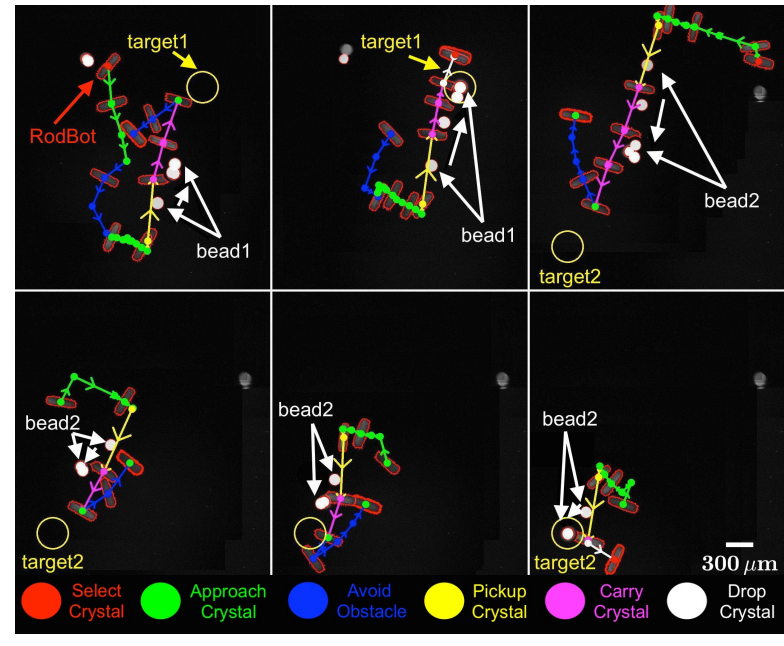

Fig. 5. A series of bead carry attempts are presented. The robot and crystal positions are superposed in one image, and to be able to track which behavior the robot is executing at any point color coded arrows are provided. First a crystal is selected and the extraction is attempted switching between various behaviors; however, the crystal is dropped prematurely (a). As the behavioral planner suggests, the system returns to earlier behaviors, picks up the bead, drops it at the target, and starts selecting another bead (b). For this trial the other bead is selected, and is carried to the target in 4 attempts (c-f).

our operational range and their sizes are comparable to average crystal sizes. Several beads and a RodBot are randomly placed in a circle of approximate diameter $5 \mathrm{~mm}$ around the center of a round glass dish of $30 \mathrm{~mm}$ diameter. The reactive behavioral planner, explained in section II-C, is modified to randomly select a drop area after each successful carry, and executed repeatedly. By randomly changing the drop zone the human factor is eliminated from the test setup, and the experiments continued until a hardware or a software failure occurs. Hundreds of successful extractions are performed, giving us the opportunity to conduct a robust statistical analysis of the system as a whole. In addition, throughout a trial, solution density and viscosity changes as it evaporates over the time. With this procedure, the system is implicitly evaluated under various medium conditions.

During the repeated trial, the following are recorded: the total number of beads carried to the target $N_{t g}$; the total number of beads detected in all the taken images $N_{i m}$; the distance $d_{i}$ for each extraction task $i$ defined as the distance between the initial bead position and the drop-zone; the time $t_{i}$ for each extraction task $i$ defined as the time elapsed from the successful completion of the carry $i-1$ until the successful completion of the carry $i$ for $i>1$; and the total time $T$ for extracting $N_{t g}$ beads. An average bead exposure time $\bar{t}_{\text {exp }}$ per extraction is calculated using $\frac{N_{i m} \cdot e}{N_{t g}}$, where $e$ is the average UV exposure time per image. The average extraction time for a single extraction $\overline{t_{e}}$ is calculated using $\frac{T}{N_{t g}}$. The average distance per extraction $\overline{d_{e}}$ is calculated using $\frac{1}{N_{t g}} \sum_{i=1}^{N_{t g}} d_{i}$. The average time per carry $\overline{t_{c}}$ is calculated using $\frac{1}{N_{t g}} \sum_{i=1}^{N_{t g}} t_{i}$. The results are presented in table I.
TABLE I

RESULTS

\begin{tabular}{|c|c|}
\hline$N_{t g}$ & 351 \\
\hline$T$ & $836 \mathrm{~min} \approx 14 \mathrm{~h}$ \\
\hline$\overline{t_{e}}$ & $2.3803 \mathrm{~min}$ \\
\hline$e$ & $0.26 \pm 0.003 \mathrm{sec}$ \\
\hline $\bar{t}_{e x p}$ & $10.8913 \mathrm{sec}$ \\
\hline$\overline{d_{e}}$ & $2.0645 \pm 0.6752 \mathrm{~mm}$ \\
\hline$\overline{t_{c}}$ & $2.3872 \pm 1.6347 \mathrm{~min}$ \\
\hline
\end{tabular}

Overall, the system ran for a total of 14 hours $(T)$ to collect a total of 351 beads (2.38 mins per collection). Comparing this to 4 hours for 100 crystals by an experienced operator (2.4 mins per collection) [2] the system operates on par with an experienced human. The mapping and the device protection behaviors are potentially time consuming ones and can each take up to 7-8 minutes. During the course of our 14hour experiment, the mapping behavior took over the control 23 times, and the device protection behavior did so 5 times. Since the $\overline{t_{e}}$ is computed using the cumulative data, the time consumed for these two behaviors is shared by all 351 task executions. However, $\overline{t_{c}}$ is computed using the timing for each carry and only a minority of the executions include the time for the two behaviors. When we recalculate $\overline{t_{c}}$ eliminating the 23 longest timings we get $2.0636 \pm 1.0112 \mathrm{mins}$ which represents a $15 \%$ improvement over experienced human performance. This confirms our conclusion. Another important detail is that the average distance per extraction with $2.0645 \mathrm{~mm}$ is considerably larger than the distances encountered during the normal crystal harvesting processes. Crystallization typically happens in droplets with diameters less than $2 \mathrm{~mm}$. Moreover, the average crystal exposure time $\bar{C}_{\text {exp }}$ is checked to ensure that the UV exposure per crystal per extraction does not exceed the limit of 100 seconds introduced in section III-A to prevent structural crystal damage. Our value of approximately 11 seconds is well within the limit.

\section{Discussion AND CONCLUSION}

Since accomplishment of our task requires meeting multiple objectives in a robust way, we propose a behaviorbased reactive planner as a compromise between planning and control. This can be viewed as a planner that divides the task at hand into several easy-to-program controllers called behaviors: approach, pickup, carry, and drop, and alternates between these as needed. This way, instead of planning for the entire harvesting process at once, the execution plan is spontaneously generated on the run, and the appropriate controller is executed as required. The primary advantage of this is that it gives the system the flexibility to fail and the means to recover from failure. Moreover, the decisions as to which behavior should take over the control are based on comparative thresholds. This relieves the need for precise measurements making the system more resilient to errors. Taking advantage of this, we manage to perform a delicate manipulation task while taking an image every 2 seconds.

This is the first automated crystal harvesting platform reported in the literature, and it has demonstrated its capability 
by harvesting over 350 crystal emulators at the same rate as an experienced human operator performing the process manually. Moreover, the test workspace covered, distances traveled, and number of collections per execution all exceed human benchmarks, making our time comparison metric unrealistically stringent. Further improvements to the system behaviors and hardware setup will continue to improve performance. For example, slight mechanical misalignment between the imaging system and the XY stage caused the workspace to leave focus, which can be improved by incorporating autofocusing. Future work will focus on integrating this platform into a complete crystallography workflow.

\section{REFERENCES}

[1] J. Drenth, Principles of Protein X-ray Crystallography. SpringerVerlag, 2007.

[2] M. C. Deller and B. Rupp, "Approaches to automated protein crystal harvesting," Acta Crystallogr. Sect. F, vol. 70, no. 2, pp. 133-155, 2014.

[3] W. Chang and D. Sretavan, "Microtechnology in medicine: the emergence of surgical microdevices," Clin. Neurosurg., vol. 54, pp. 137-47, 2007.

[4] B. Hannaford, J. Hewitt, T. Maneewarn, S. Venema, M. Appleby, and R. Ehresman, "Telerobotic remote handling of protein crystals," in IEEE Int Conf Robot, vol. 2, pp. 1010-1015 vol.2, Apr 1997.

[5] M. Gittleman, R. Hunter, N. Smith, and A. Johnston, "Robotic harvesting of solids from fluids," July 9 2002. US Patent 6,417,007.

[6] K. Ohara, K. Ohba, T. Tanikawa, M. Hiraki, S. Wakatsuki, M. Mizukawa, and K. Tanie, "Automated micro manipulation system with protein crystal," in IEEE Int Symp Micro-nanomech, pp. 301-306, Oct 2004.

[7] K. Ohara, K. Ohba, T. Tanikawa, M. Hiraki, S. Wakatsuki, and M. Mizukawa, "Hands free micro operation for protein crystal analysis," in IEEE/RSJ Inter. Conf. Intelligent Rob. Sys., vol. 2, pp. 17281733, 2004.

[8] C. G. Roessler, A. Kuczewski, R. Stearns, R. Ellson, J. Olechno, A. M. Orville, M. Allaire, A. S. Soares, and A. Héroux, "Acoustic methods for high-throughput protein crystal mounting at next-generation macromolecular crystallographic beamlines," J. Synchrotron Radiat., vol. 20, pp. 805-808, Sep 2013.

[9] A. Wagner, R. Duman, B. Stevens, and A. Ward, "Microcrystal manipulation with laser tweezers," Acta Crystallogr. Sect. D, vol. 69, pp. 1297-1302, Jul 2013.

[10] H.-W. Tung, K. E. Peyer, D. F. Sargent, and B. J. Nelson, "Noncontact manipulation using a transversely magnetized rolling robot," Appl. Phys. Lett., vol. 103, p. 114101, September 2013.

[11] H.-W. Tung, D. F. Sargent, and B. J. Nelson, "Protein crystal harvesting using the rodbot - a wireless, mobile microrobot," J. Appl. Crystallogr., vol. 4, pp. 692-700, 2014.

[12] C. A. Cumbaa, A. Lauricella, N. Fehrman, C. Veatch, R. Collins, J. Luft, G. DeTitta, and I. Jurisica, "Automatic classification of sub-microlitre protein-crystallization trials in 1536-well plates," Acta Crystallogr. Sect. D, vol. 59, no. 9, pp. 1619-1627, 2003.

[13] R. Liu, Y. Freund, and G. Spraggon, "Image-based crystal detection: a machine-learning approach," Acta Crystallogr. Sect. D, vol. 64, no. 12, pp. 1187-1195, 2008.

[14] J. T. Ng, C. Dekker, M. Kroemer, M. Osborne, and F. von Delft, "Using textons to rank crystallization droplets by the likely presence of crystals," Acta Crystallogr. Sect. D, vol. 70, no. 10, pp. 2702-2718, 2014.

[15] S. Stepanov, M. Hilgart, D. W. Yoder, O. Makarov, M. Becker, R. Sanishvili, C. M. Ogata, N. Venugopalan, D. Aragão, M. Caffrey, J. L. Smith, and R. F. Fischetti, "Fast fluorescence techniques for crystallography beamlines," J. Appl. Crystallogr., vol. 44, no. 4, pp. 772-778, 2011.

[16] K. Dierks, A. Meyer, D. Oberthür, G. Rapp, H. Einspahr, and C. Betzel, "Efficient UV detection of protein crystals enabled by fluorescence excitation at wavelengths longer than 300nm," Acta Crystallogr. Sect. $F$, vol. 66, no. 4, pp. 478-484, 2010.
[17] D. J. Kissick, C. M. Dettmar, M. Becker, A. M. Mulichak, V. Cherezov, S. L. Ginell, K. P. Battaile, L. J. Keefe, R. F. Fischetti, and G. J. Simpson, "Towards protein-crystal centering using second-harmonic generation (SHG) microscopy," Acta Crystallogr. Sect. D, vol. 69, no. 5, pp. 843-851, 2013.

[18] A. Banerjee and S. Gupta, "Research in automated planning and control for micromanipulation," IEEE Trans. Autom. Sci. Eng., vol. 10, no. 3, pp. 485-495, 2013.

[19] A. Thakur, S. Chowdhury, P. Švec, C. Wang, W. Losert, and S. K. Gupta, "Indirect pushing based automated micromanipulation of biological cells using optical tweezers," Int. J. Rob. Res., vol. 33, pp. 1098-1111, July 2014.

[20] R. J. Firby, "An investigation into reactive planning in complex domains.," in $A A A I$ (K. D. Forbus and H. E. Shrobe, eds.), pp. 202 206, Morgan Kaufmann, 1987.

[21] R. S. Pieters, H.-W. Tung, S. Charreyron, S. F. Sargent, and B. J. Nelson, "Rodbot: a rolling microrobot for micromanipulation," in Proc. IEEE Int. Conf. Robotics and Automation, pp. 4042-4047, 2015.

[22] S. Schuerle, S. Erni, M. Flink, B. Kratochvil, and B. Nelson, "Threedimensional magnetic manipulation of micro- and nanostructures for applications in life sciences," IEEE Trans. on Magnetics, vol. 49, no. 1, pp. 321-330, 2013.

[23] B. E. Kratochvil, M. P. Kummer, S. Erni, R. Borer, D. R. Frutiger, S. Schürle, and B. J. Nelson, MiniMag: A Hemispherical Electromagnetic System for 5-DOF Wireless Micromanipulation, pp. 317-329. Berlin, Heidelberg: Springer Berlin Heidelberg, 2014.

[24] A. J. Petruska and B. J. Nelson, "Minimum bounds on the number of electromagnets required for remote magnetic manipulation," IEEE Trans. Robot., vol. 31, no. 3, pp. 714-722, 2015.

[25] D. Voet and J. G. Voet, Biochemistry, 2nd ed. John Wiley \& Sons, 1995.

[26] J. R. Lakowicz, Principles of Fluorescence Spectroscopy. Springer, 1999.

[27] R. A. Judge, K. Swift, and C. González, "An ultraviolet fluorescencebased method for identifying and distinguishing protein crystals," Acta Crystallogr. Sect. D, vol. 61, no. 1, pp. 60-66, 2005.

[28] G. Bradski, "The OpenCV library," Dr. Dobb's J. Soft Tools, 2000.

[29] R. Brooks, "A robust layered control system for a mobile robot," IEEE J. Robot. Autom., vol. 2, pp. 14-23, Mar 1986.

[30] R. Brooks, "Intelligence without representation," Artif. Intell., vol. 47, pp. 139-159, 1991.

[31] A. Linson, C. Dobbyn, G. E. Lewis, and R. Laney, "A subsumption agent for collaborative free improvisation," Compt Music J, 2016.

[32] E. Oland, T. S. Andersen, and R. Kristiansen, "Subsumption architecture applied to flight control using composite rotations," Automatica, vol. 69, pp. 195-200, 2016.

[33] G. Greenwood, M. Podhradsky, J. Gallagher, and E. Matson, "A multiagent system for autonomous adaptive control of a flapping-wing micro air vehicle," in IEEE Symp Series Comp Intel, pp. 1073-1080, Dec 2015.

[34] H. Saito, R. Amano, N. Moriyama, K. Kobayashi, and K. Watanabe, "Emergency obstacle avoidance module for mobile robots using a laser range finder," in Proc SICE Ann Conf, pp. 348-353, IEEE, 2013.

[35] M. Da Lio, F. Biral, E. Bertolazzi, M. Galvani, P. Bosetti, D. Windridge, A. Saroldi, and F. Tango, "Artificial co-drivers as a universal enabling technology for future intelligent vehicles and transportation systems," IEEE Trans Intel Trans Sys, vol. 16, no. 1, pp. 244-263, 2015.

[36] O. Khatib, "Real-time obstacle avoidance for manipulators and mobile robots," Int. J. Rob. Res., vol. 5, pp. 90-98, Apr. 1986.

[37] B. Zeydan and H. L. Akın, "Market-driven multi-agent collaboration for extinguishing fires in the robocup rescue simulation domain," in CSW-2011 Comp. Sci. Student Workshop, p. 5, 2011.

[38] F. Wien, A. J. Miles, J. G. Lees, S. Vrønning Hoffmann, and B. A Wallace, "VUV irradiation effects on proteins in high-flux synchrotron radiation circular dichroism spectroscopy," J. Synchrotron Radiat., vol. 12, pp. 517-523, Jul 2005.

[39] X. Vernede, B. Lavault, J. Ohana, D. Nurizzo, J. Joly, L. Jacquamet, F. Felisaz, F. Cipriani, and D. Bourgeois, "UV laser-excited fluorescence as a tool for the visualization of protein crystals mounted in loops," Acta Crystallogr. Sect. D, vol. 62, pp. 253-261, Mar 2006. 